\title{
Deficiencies of combined rank tests
}

\author{
Willem Albers \\ Department of Applied Mathematics, Twente University of Technology, Enschede, Netherlands
}

Received 20 December 1989; revised manuscript received 19 November 1990

\begin{abstract}
One- and two sample problems are considered which are divided into subproblems, for each of which a separate rank statistic is obtained. The best combination of these statistics is then compared to the ordinary undivided rank statistic. One easily sees that, under natural conditions, splitting causes no first order efficiency loss. Hence it becomes interesting to derive second order results. The required methods are rather technical but fortunately much can be built on earlier work. The results are simple and quite encouraging: enlarging the number of subgroups by one typically costs about one additional observation. A small simulation study confirms these results.
\end{abstract}

AMS Subject Classification: $62 \mathrm{G} 10,62 \mathrm{G} 20$.

Key words and phrases: One-sample problem; two-sample problem; asymptotic efficiency; second order efficiency; asymptotic expansions.

\section{Introduction}

Consider a testing problem in which the total sample has been divided into $r$ $(r \geqslant 2)$ subsamples. For each of these subsamples a suitable statistic $T_{v}, v=1, \ldots, r$, is available. In this situation it is interesting to find the best combination $T^{*}$ of these $T_{1}$, and to compare its performance to that of the standard statistic $T$ which would have been used for the total sample if it were undivided. If $T$ turns out to be only marginally better than $T^{*}$, it does not seem very worthwhile to reconstruct $T$, even if the original observations are still available, and not merely the $T_{v}, v-1, \ldots, r$.

This subject and related ones have received considerable attention in the literature. For earlier work we refer to van Zwet and Oosterhoff (1967) and Lehmann (1975), p. 281. In the present paper we shall concentrate on one-and two-sample rank tests. There are several reasons for this special interest. To begin with, Albers and Akritas (1987) recently presented a new class of rank tests under random censor ship, based on the idea of ranking the uncensored and the censored observations separately and subsequently combining the resulting rank statistics. They already

Correspondence to: Prof. Willem Albers, Dept. of Applied Mathematics, Twente University of Technology, P.O. Box 217, 7500 AE Enschede, Netherlands. 
demonstrated that the efficiency loss due to this splitting is asymptotically negligible, and suggest that it will even be $\mathrm{O}\left(N^{-1 / 2}\right)$, where $N$ is the total sample size. But it would be nice to obtain more precise results on the effect of splitting. A second application which calls for a detailed comparison of combined and ordinary rank statistics, is the construction of nonparametric two-stage procedures, see Albers (1991, 1992).

A third and more pragmatic reason is the fact that the basic techniques and results for such finer comparisons are readily available from the paper by Albers, Bickel and van Zwet (1976) and Bickel and van Zwet (1978). These papers will be denoted in the sequel by $A B Z$ and $B Z$, respectively. As a lot of material is taken from $A B Z$ and $B Z$, readers interested in technical details are advised to consult these papers for the necessary background.

After this motivation, let us briefly recall why the combined statistic $T^{*}$ is first order equivalent to $T$ (also cf. Albers and Akritas (1987), p. 650). Details and conditions will be postponed to later sections when we deal with the second order terms. Suppose that for testing $\mathbf{H}_{0}: \theta=0$ against $\mathbf{H}_{1}: \theta>0$ the statistics $T_{v}$ are asymptotically normal with means $\theta \mu_{1}$ and variances $\sigma_{v}^{2}, v=1, \ldots, r$. We shall denote this as:

$$
T_{v} \text { is } \mathrm{AN}\left(\theta \mu_{v}, \sigma_{v}^{2}\right), \quad v=1, \ldots, r \text {. }
$$

A straightforward computation reveals that, if we want to maximize the asymptotic power, the best combination $T^{*}$ of these $T_{v}$ for this purpose is proportional to $\sum_{v=1}^{r} \mu_{v} T_{v} / \sigma_{v}^{2}$, which is, in its turn,

$$
\operatorname{AN}\left(\theta \sum_{v=1}^{r} \frac{\mu_{v}^{2}}{\sigma_{v}^{2}}, \sum_{v=1}^{r} \frac{\mu_{v}^{2}}{\sigma_{v}^{2}}\right) .
$$

For onc- and two-sample rank statistics we typically have that $\mu_{v}=N_{v} \mu$ and $\sigma_{v}^{2}=$ $N_{r}, \sigma^{2}$, where $N_{v}$ is the size of the $v$-th subsample.

Consequently, in this situation we can simply let $T^{*}=\sum_{v=1}^{r} T_{v}$, which is $\operatorname{AN}\left(\theta \mu \sum_{v=1}^{r} N_{v}, \sigma^{2} \sum_{v=1}^{r} N_{v}\right)$. However, as $\sum_{v=1}^{r} N_{v}=N$, this is nothing but the asymptotic distribution of the total sample statistic $T$. Hence $T^{*}$ and $T$ are first order equivalent. It should be noted that in the two-sample case this result holds true only under the additional requirement that the two samples, of sizes say $m$ and $n=N-m$, are split in such a way that the subsample sizes $m_{v}$ and $n_{v}$ satisfy

$$
\frac{m_{v}}{m}=\frac{n_{v}}{n}=\frac{N_{v}}{N}, \quad v=1, \ldots, r,
$$

again to the first order. This because $\mu_{v}$ and $\sigma_{v}^{2}$ depend on $v$ through $m_{v} n_{v} / N_{v}$, which permits an expression like $\mu_{v}=N_{v} \mu$ only under (1.1).

On the other hand, it is quite fair to impose (1.1): it is intuitively clear that we only stand a chance of competing successfully through our combined statistic $T^{*}$ with the usual $T$ if the balance of the sample sizes has been preserved under the splitting.

Now that we have established some belief in the first order equivalence of $T^{*}$ and 
$T$, it becomes interesting to try a bit harder and to move on to second order comparisons in the sense of Hodges and Lehmann (1970). The idea briefly is as follows. If $T$ is based on $N$ observations, then the test based on $T^{*}$ requires $k=k_{N}$ observations to match the power attained by the test based on $T$. First order equivalence of $T^{*}$ and $T$ means that $e_{N}=N / k_{N} \rightarrow 1$ as $N \rightarrow \infty$. Hence the so-called deficiency $d_{N}=k_{N}-N=N\left(e_{N}{ }^{1}-1\right)$ satisfies $d_{N}=\mathrm{o}(N)$, which can still vary considerably. Second order comparisons are aimed at determining the actual order of $d_{N}$ and preferably the precise behavior of its leading term. Required for such comparisons are not mere asymptotic normality results, but asymptotic expansions to $o\left(N^{-1}\right)$, which typically are much harder to get. An idea of the complications involved can be obtained by consulting the aforementioned papers $\mathrm{ABZ}$ and $\mathrm{BZ}$, which cover the oneand the two-sample case, respectively.

In view of the rather extreme technicality of these two papers, it might be feared that efforts to combine such results for various subgroups, will lead to expressions which are truly awful. Fortunately, this is absolutely not the case. By first looking at the classical situation of sums of independent random variables, we derive some simple criteria to determine which of the multitude of second order terms will cause differences between $T^{*}$ and $T$, and which will not. It turns out that the latter group forms an overwhelming majority. (But the first group is not empty!) To give an idea of the simplicity of the results, we single out the case of Wilcoxon's two-sample test against logistic location alternatives. We shall prove that the deficiency $d_{N}$ in this example tends to the finite limit

$$
d=r-1 \text {. }
$$

In other words, the penalty to be paid for dividing the total sample into subgroups asymptotically equals one additional observation for each additional group. Note especially that this result is independent of the size of the test and the particular alternative considered.

In the above we have sketched what we want to do, and why and how we want to do it. It remains to list the contents of the other sections. In Section 2 we shall collect the necessary results on convolutions of expansions. In the next section we apply these results and the relevant ones from ABZ to the one-sample case. In Section 4 we deal, using $B Z$, with the additional complications that arise in the twosample case. Finally, in Section 5, we present by way of illustration a small simulation study for the case of the one-sample Wilcoxon test against logistic alternatives. The numerical results agree very well with the predicted theoretical values.

\section{Convolutions of expansions}

Let $T$ be a statistic with a continuous distribution function (df). Consider a standardized version

$$
S=\frac{T-\zeta}{\beta},
$$


such that the df $G$ of $S$ can be approximated by $\Phi(x-\eta)$, where $\Phi$ is the standard normal $\mathrm{df}$. There may seem to be some redundancy here: if $\zeta$ is replaced by $\zeta+\beta \eta$ in (2.1), $G$ is approximated by $\Phi$ itself. However, in applications $\eta$ will typically vary with the unknown underlying distribution, which should of course not be the case for $\zeta$.

To arrive at a refinement of the above, we observe that the normal approximation amounts to a result like $\sup _{x}|G(x)-\Phi(x-\eta)| \leqslant \delta$, in which for given size $N$ of the underlying sample $\delta$ is a positive and typically small constant, while moreover $\delta \rightarrow 0$ as we let $N \rightarrow \infty$. Often it is desirable to sharpen such a result to one in which $\delta$ is not merely o(1), but e.g. $o\left(N^{-1}\right)$. Typically this entails replacement of the first order normal approximation $\Phi(\tilde{x})$, where $\tilde{x}=x-\eta$, by a more sophisticated approximation $\tilde{G}(\tilde{x})$, which has $\Phi(\tilde{x})$ as its leading term. A common approach to building classes of such $\tilde{G}(\tilde{x})$ proceeds as follows. Let $\phi$ be the standard normal density and let $\phi^{(k)}, k=1,2, \ldots$, be the $k$-th derivative of $\phi=\phi^{(0)}$. Then the Hermite polynomial of degree $k$ is defined through

$$
H_{k}(x)=\frac{(-1)^{k} \phi^{(k)}(x)}{\phi(x)}, \quad k=0,1,2, \ldots
$$

We thus have $H_{0}(x)=1, H_{1}(x)=x, H_{2}(x)=x^{2}-1, H_{3}(x)=x^{3}-3 x$, and so $o$.

Now we can construct more sophisticated approximations $\tilde{G}(\tilde{x})$ for the $\mathrm{df} G$ of $S$ from (2.1) such that for some positive constant $\delta$

$$
\sup _{x}|G(x)-\tilde{G}(\tilde{X})| \leqslant \delta,
$$

where $\tilde{x}=x-\eta$ and

$$
\tilde{G}(x)=\Phi(x)+\phi(x) \sum_{k=0}^{p} b_{k} H_{k}(x) .
$$

Again the idea is of course to select the coefficients $b_{k}$ in such a way that $\delta$ is as small as possible for given $p$. Moreover the statistic $T$, and hence $\zeta, \beta, \eta, \delta$ and the $b_{k}$, will usually depend on the size $N$ of the underlying sample and not only $\delta$, but also $b_{k}$ will tend to zero as $N \rightarrow \infty$. But at this point the purely formal result in $(2.3)$ and (2.4) suffices. We shall merely assume for convenience that all $b_{k}$ satisfy $\left|b_{k}\right| \leqslant 1$. Hence in other words, only the general structure of the approximation $\tilde{G}$ has been determined so far and a wide variety of possible choices remains. Later on, in Sections 3 and 4, we will consider explicit choices, resulting in unique $\tilde{G}$. But the additional details involved would at this stage merely obscure what is going on in general.

Next suppose we have independent statistics $T_{v}, v=1, \ldots, r$, for each of which the above exposition holds. Hence we encounter $S_{v}, \zeta_{v}, \beta_{v}, G_{v}, \eta_{v}, \tilde{G}_{v}, b_{k v}, \delta_{v}$ and $x_{v}=$ $x-\eta_{v}$. Typically the same accuracy will be required for each $v$, so there is no need to let $p$ depend on $v$ as well. From the introduction it is clear that we are particularly interested in $T^{*}=\sum_{r^{*}=1}^{r} T_{v}$. As its standardized version we obtain 


$$
S^{*}=\frac{T^{*}-\sum_{v^{\prime}=1}^{r} \zeta_{v}}{\left(\sum_{v=1}^{r} \beta_{v}^{2}\right)^{1 / 2}}=\sum_{v=1}^{r} \gamma_{v} S_{v},
$$

where $\gamma_{v}$ by definition equals $\beta_{v} /\left(\sum_{g=1}^{r} \beta_{g}^{2}\right)^{1 / 2}$ and satisfies

$$
\sum_{v=1}^{r} \gamma_{v}^{2}=1
$$

Our first goal now is to derive an approximation for the df of $S^{*}$ in (2.5) from the approximations $\tilde{G}_{v}\left(x_{v}\right)$ for $G(x), v=1, \ldots, r$. The result is presented in the following lemma. Bearing in mind that in our intended applications the $b_{k v}$ will tend to zero, we relegate all terms involving three or more factors $b_{k v}$ to the remainder.

Lemma 2.1. Suppose $S_{v}, v=1, \ldots, r$, are independent statistics with continuous df's $G_{1}$, admitting approximations $\tilde{G}_{v}$ satisfying (2.3) and $(2.4)$ for certain $p, b_{k v}$ with $\left|b_{k v}\right| \leqslant 1, \delta_{v}$ and $x_{v}=x-\eta_{v}$. Then the df $G^{*}$ of $S^{*}$ from (2.5) satisfies, for certain nonnegative constants $C_{1}$ and $C_{2}$,

$$
\begin{aligned}
& \sup _{*}\left|G^{*}(x)-\tilde{G}^{*}\left(x^{*}\right)\right| \\
& \leqslant\left(\sum_{v=1}^{r} \delta_{k}\right)\left(1+C_{1} \sum_{k=1}^{r} \sum_{k=0}^{p}\left|b_{k v}\right|\right)+C_{2} \sum_{v=1}^{r}\left(\sum_{k=0}^{p}\left|b_{k v}\right|\right)^{3},
\end{aligned}
$$

where $x^{*}=x-\sum_{i=1}^{r} \gamma_{v} \eta_{v}$ and

$$
\begin{aligned}
& \widetilde{G}^{*}(x)=\Phi(x)+\phi(x)\left\lfloor\sum_{k=0}^{p} \sum_{v=1}^{r} b_{k v} \gamma_{v}^{k+1} H_{k}(x)\right. \\
&\left.-\sum_{k=0 l=0}^{p} \sum_{v=1}^{p} \sum_{k=1}^{r} b_{k v} b_{k g} \gamma_{v}^{k+1} \gamma_{k}^{l+1} H_{k+1+1}(x)\right\rfloor .
\end{aligned}
$$

Proof. First we consider the case $r=2$. Since $S^{*}=\gamma_{1} S_{1}+\gamma_{2} S_{2}$ we clearly have that $G^{*}(z)=\int G_{2}\left((z-x) / \gamma_{2}\right) \mathrm{d} G_{1}\left(x / \gamma_{1}\right)$. Using (2.3), (2.4) and the fact that the $G_{v}$ and $\tilde{G}_{1,}$ are of bounded variation and continuous (cf. Widder (1966), Chapter 1), we obtain for all $z$,

$$
\begin{aligned}
& \left|G^{*}(z)-\right| \tilde{G}_{2}\left(\frac{z-x}{\gamma_{2}}-\eta_{2}\right) \mathrm{d} \tilde{G}_{1}\left(\frac{x}{\gamma_{1}}-\eta_{1}\right) \mid \\
& \leqslant \|\left(G_{2}\left(\frac{z-x}{\gamma_{2}}\right)-\tilde{G}_{2}\left(\frac{z-x}{\gamma_{2}}-\eta_{2}\right)\right) \mathrm{d} G_{1}\left(\frac{x}{\gamma_{1}}\right) \mid \\
& +|| \tilde{G}_{2}\left(\frac{z-x}{\gamma_{2}}-\eta_{2}\right) \mathrm{d}\left(G_{1}\left(\frac{x}{\gamma_{1}}\right)-\tilde{G}_{1}\left(\frac{x}{\gamma_{1}}-\eta_{1}\right)\right) \mid \\
& \leqslant \delta_{2}+\left|\dot{\int}\left(G_{1}\left(\frac{x}{\gamma_{1}}\right)-\tilde{G}_{1}\left(\frac{x}{\gamma_{1}}-\eta_{1}\right)\right) \mathrm{d} \tilde{G}_{2}\left(\frac{z-x}{\gamma_{2}}-\eta_{2}\right)\right| \\
& \leqslant \delta_{2}+\delta_{1}\left(1+C \sum_{k=0}^{p}\left|b_{k 2}\right|\right) \text {, }
\end{aligned}
$$


for some positive constant $C$. Obviously, the upper bound remains valid as such if the indices 1 and 2 are interchanged. Averaging these two possibilities, we arrive at the symmetrical expression corresponding to the first two terms on the right-hand side of (2.7). (The third term will only be needed for $r>2$.)

Hence it remains to show that the approximation

$$
\int \tilde{G}\left(\frac{z-x}{\gamma_{2}}-\eta_{2}\right) \mathrm{d} \tilde{G}_{1}\left(\frac{x}{\gamma_{1}}-\eta_{1}\right)
$$

from (2.9) for the df $G^{*}$ equals $\hat{G}^{*}\left(x^{*}\right)$ from (2.8). This follows by straightforward computation, using the fact that

$$
\int_{-\infty}^{\infty} \Phi^{(k)}\left(\frac{z-x}{\gamma_{2}}-\eta_{2}\right) \mathrm{d} \Phi^{(l)}\left(\frac{x}{\gamma_{1}}-\eta_{1}\right)=\gamma_{2}^{k} \gamma_{1}^{l} \Phi^{(k+l)}\left(z-\gamma_{1} \eta_{1}-\gamma_{2} \eta_{2}\right),
$$

for $k, l \geqslant 0$. (Note that the factor $\sum_{v=1}^{r} \gamma_{v}^{2}$ vanishes in view in (2.6).)

Alternatively, since

we have

$$
\int_{-\infty}^{\infty} \mathrm{e}^{\mathrm{i} t x} \mathrm{~d}\left(H_{k}(x) \phi(x)\right)=-(\mathrm{i} t)^{k+1} \mathrm{e}^{-t^{2} / 2},
$$

and

$$
\tilde{\varrho}_{v}(t)=\int_{-\infty}^{\infty} \mathrm{e}^{\mathrm{i} / x} \mathrm{~d} \tilde{G}_{v}(x)=\mathrm{e}^{-t^{2} / 2}\left\{1-\sum_{k=0}^{p} b_{k v}(\mathrm{i} t)^{k+1}\right\},
$$

$$
\prod_{v} \exp \left(\mathrm{i} t \gamma_{\nu} \eta_{v}\right) \tilde{\varrho}_{v}\left(\gamma_{v} t\right)=\exp \left(\mathrm{i} t \sum_{v} \gamma_{v} \eta_{v}-\frac{1}{2} t^{2}\right) \prod_{v}\left\{1-\sum_{k=0}^{p} b_{k v}\left(\mathrm{i} \gamma_{v} t\right)^{k+1}\right\}
$$

which also readily produces the desired result. Moreover, the generalization to $r>2$ is now immediate: Fourier inversion of the first three terms in this product still leads to $\widetilde{G}^{*}\left(x^{*}\right)$. The difference is that for $r>2$ a number of terms remain. However, each of these terms consists of a certain derivalive of $\phi$, which is bounded, times a coefficient containing at least three $b_{k v}$. Since all $\left|b_{k v}\right| \leqslant 1$, these coefficients are all of order $\sum_{v=1}^{r}\left(\sum_{k=0}^{p}\left|b_{k v}\right|\right)^{3}$. Hence by adding the third remainder term in $(2.7)$, this result becomes valid for $r>2$ as well.

The next step will be the application of this result to our splitted sample problem. Hence $T$, and thus $S$ from (2.1), now will be a statistic based on a total sample of size $N$, designed to test a hypothesis about an unknown parameter $\theta$, for example $\mathrm{H}_{0}: \theta=0$ against $\mathrm{H}_{1}: \theta>0$. Our $T_{v}, v=1, \ldots, r$, are test statistics for the same problem, the only difference being that these statistics are based on samples of sizes $N_{v}$, $v=1, \ldots, r$. To allow the splitting interpretation, we obviously require that $\sum_{v=1}^{r} N_{v}=$ $N$. Let $\tilde{\gamma}_{\nu}=\left(N_{\nu} / N\right)^{1 / 2}$, then (cf. (2.6))

$$
\sum_{v=1}^{r} \tilde{\gamma}_{v}^{2}=1
$$

Now it becomes useful to denote explicitly the dependence of $\eta, b_{k}$ and $\delta$ from 
(2.3) and (2.4) on $N$ and $\theta$ by writing $\eta=\eta(\theta, N), b_{k}=b_{k}(\theta, N)$ and $\delta=\delta(N)$. We then immediately have for $\eta_{v}, b_{k v}$ and $\delta_{v}$ the relations

$$
\eta_{v}=\eta\left(\theta, N_{v}\right), \quad b_{k v}=b_{k}\left(\theta, N_{v}\right), \quad \delta_{v}=\delta\left(N_{v}\right)
$$

In this way we have also established the connection between the approximation $\tilde{G}(\tilde{x})$ from (2.3) and (2.4) for the df $G(x)$ of the standardized total statistic from (2.1) on one hand, and the approximation $\tilde{G}^{*}\left(x^{*}\right)$ from (2.7) and $(2.8)$ for the $\mathrm{df} G^{*}(x)$ of the standardized combined statistic from (2.5) on the other.

However, this connection does not seem to be very fruitful: even under (2.12), the approximations (2.8) and (2.4) look quite different and the supposed similarity of $T^{*}$ and $T$ is not visible. Therefore we shall present in the following lemma two simple conditions under which similarities between $\tilde{G}^{*}$ and $\tilde{G}$ do become apparent. Typically the first of these conditions is meant for terms of order $N^{-1}$ and the second for those of order $N^{-1 / 2}$.

Lemma 2.2. (i) Suppose that for a certain $k_{0}$,

$$
b_{\left.k_{i}\right)}\left(\theta, N_{v}\right)=\gamma_{v}^{\left(1-k_{0}\right)} b_{k_{i}}(\theta, N)
$$

with $\gamma_{v}$ as defined at (2.6).

(ii) Suppose that for certain $k_{0}$ and $l_{0}$,

$$
b_{k_{11}+l_{11}+1}\left(\theta, N_{v}\right)=-\left\{1-\frac{1}{2} \delta\left(k_{0}, l_{0}\right)\right\} b_{k_{0}}\left(\theta, N_{v}\right) b_{l_{0}}\left(\theta, N_{v}\right),
$$

where both $b_{k_{1}}\left(0, N_{v}\right)$ and $b_{l_{0}}\left(0, N_{v}\right)$ satisfy $(2.13)$ and $\delta(\cdot, \cdot)$ stands for Kronecker's delta.

Then we have that under (i) the coefficients of $H_{k_{1}}$ in $\tilde{G}$ from (2.4) and in $\tilde{G}^{*}$ from (2.8) agree. Under (ii) this holds for $H_{k_{11}+l_{11}+1}$.

Proof. (i) Under (2.13) we obtain that

$$
\sum_{v-1}^{r} b_{k_{0}}\left(\theta, N_{v}\right) \gamma_{v}^{k_{v}+1}=b_{k_{0}}(\theta, N) \sum_{v-1}^{r} \gamma_{v}^{2}=b_{k_{v}}(\theta, N),
$$

in view of (2.6).

(ii) Under (2.13) and (2.14) we obtain for $k_{0} \neq l_{0}$ that the coefficient $H_{k_{0}+l_{1}+1}$ in $\tilde{G}$ equals

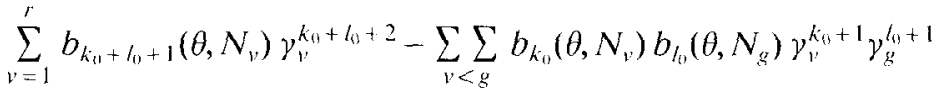

$$
\begin{aligned}
& -\sum_{v<g} \sum_{l_{0}}\left(\theta, N_{v}\right) b_{k_{0}}\left(\theta, N_{g}\right) \gamma_{v^{\prime \prime}}^{l_{1}+1} \gamma_{g}^{k_{0}+1} \\
& =-b_{k_{0}}(\theta, N) b_{l_{0}}(\theta, N)\left\{\sum_{v} \gamma_{v}^{4}+2 \sum_{v<g} \sum_{v} \gamma_{v}^{2} \gamma_{g}^{2}\right\} \\
& =-b_{k_{0}}(\theta, N) b_{l_{0}}(\theta, N)=b_{k_{0}+l_{11}+1}(\theta, N) \text {. }
\end{aligned}
$$

For $k_{0}=l_{0}$, the proof is virtually identical. 
Remark. A generalization is the following: suppose $b_{k}\left(\theta, N_{v}\right)$ does not satisfy the conditions above, but can be expressed as $\sum_{j=1}^{q_{k}} b_{k}^{(j)}\left(\theta, N_{v}\right)$. Then the lemma can be applied separately to each of the summands for which the conditions do hold. As this generalization is rather trivial, we will not prove it, but we shall nevertheless feel free to use it.

The fact that $T^{*}$ will resemble $T$ if $(2.13)$ or (2.14) apply to all or most coefficients involved, is quite evident. However, at first sight these conditions look rather peculiar and it is not clear why they will indeed be satisfied often. Therefore we close this section with a trivial but illuminating example, before moving on to the real applications in Sections 3 and 4.

The example we mean is concerned with the case of independent identically distributed (i.i.d.) random variables (r.v.'s) $X_{1}, \ldots, X_{N}$ from a continuous distribution function $F(x-\theta)$, together with the choice $T=\sum_{j=1}^{N} X_{j}$. Let $T_{1}$ simply be the sum of the first $N_{1}$ r.v.'s $X_{j}$, and $T_{2}$ the sum of the next $N_{2}$ r.v.'s $X_{j}$, etc. Clearly $T^{*}=\sum_{v=1}^{r} T_{v}$ is identical to $T$ in this example and hence $G^{*}=G$. A typical choice for $\tilde{G}(\tilde{x})$ is the Edgeworth expansion to order $N^{-1}$ (see Cramér (1946), p. 229). The obvious norming constants to be used are $\zeta=N \mu=N E_{0} X_{1}, \beta=N^{1 / 2} \sigma=N^{1 / 2} \sigma_{0}\left(X_{1}\right)$ and $\eta=N^{1 / 2} \theta / \sigma$, under the equally obvious assumptions $0<\sigma<\infty$ and $0 \leqslant \theta=$ $\mathrm{O}\left(N^{-1 / 2}\right)$. Then $\tilde{G}$ in (2.4) is determined by $p=5, b_{0}=b_{1}=b_{4}=0$ and

$$
b_{2}(\theta, N)=-\frac{\kappa_{3}}{6 N^{1 / 2}}, \quad b_{3}(\theta, N)=-\frac{\kappa_{4}}{24 N}, \quad b_{5}(\theta, N)=-\frac{\kappa_{3}^{2}}{72 N},
$$

where $\kappa_{3}=E_{0}\left(X_{1}-\mu\right)^{3} / \sigma^{3}, \kappa_{4}=E_{0}\left(X_{1}-\mu\right)^{4} / \sigma^{4}-3$ and thus $E_{0} X_{1}^{4}$ is assumed to be finite. In this case $\tilde{\gamma}_{v}=\gamma_{v}=\left(N_{v} / N\right)^{1 / 2}$ (cf. (2.6) and (2.11)). (Assume that $\gamma_{v} \geqslant \varepsilon>0$, $v=1, \ldots, r$, for some $\varepsilon>0$.) It follows that $b_{2}$ and $b_{3}$ from (2.15) satisfy the first condition of Lemma 2.2, while $b_{5}$ from (2.15) satisfies the second. Consequently, $\tilde{G}^{*}$ agrees with $\tilde{G}$ as far as $H_{2}, H_{3}$ and $H_{5}$ are concerned, and hence the two agree to $\operatorname{order} N^{-1}$, as the remaining terms in $\widetilde{G}^{*}$ are $O\left(N^{-3 / 2}\right)$. As finally

$$
x^{*}=x-\sum_{v=1}^{r} \gamma_{v} \eta_{v}=x-\eta \sum_{v=1}^{r} \gamma_{v}^{2}=x-\eta=\tilde{x},
$$

it follows that the two approximations $\tilde{G}(\tilde{x})$ and $\tilde{G}^{*}\left(x^{*}\right)$ coincide to the order under consideration, as should be the case in view of the fact that both approximate the same df $G^{*}=G$.

Having established that the conditions work, it remains to clarify why they work. This is most easily done by looking at the characteristic functions (chf's) again. Let $\varrho$ be the chf of $X_{1}-\mu$, then the chf $\varrho_{S}$ of $S$ equals

$$
\exp \left(N \log \varrho\left(\frac{t}{\sigma N^{1 / 2}}\right)\right)=\exp \left(\sum_{v=1}^{r} N_{v} \log \varrho\left(\frac{\nu_{v} t}{\sigma N_{v}^{1 / 2}}\right)\right) .
$$

Expanding both sides we obtain a term proportional to $N^{(1-k) / 2}(\mathrm{i} t / \sigma)^{k+1}$ on the left-hand side, and proportional to $\sum_{v=1}^{r} N_{v}^{(1-k) / 2}\left(\mathrm{i} \tilde{\gamma}_{v} t / \sigma\right)^{k+1}$ on the right-hand 
side. As $\tilde{\gamma}_{v}=\gamma_{v}=\left(N_{v} / N\right)^{1 / 2}$ and Fourier inversion relates the $(\mathrm{i} t)^{k+1}$-term to $H_{k}$, a result like (2.13) suggests itself. To explain (2.14), note that the expansion involves an exponential and that $\exp (-x)=1-x+x^{2} / 2+\mathrm{O}\left(x^{3}\right)$ for small $x$. Hence the $(\mathrm{i} t)^{k \cdot l}$-term and the $(\mathrm{i} t)^{l+1}$-term in $x$ lead to a product term involving $(\mathrm{i} t)^{k+l+2}$, which corresponds to $H_{k+1+1}$.

\section{The one-sample case}

Let $X_{1}, \ldots, X_{N}$ be i.i.d. r.v.'s with continuous df $F(x-\theta)$. Suppose that $F(-x)=$ $1-F(x)$ for all $x$. Hence under $\mathrm{H}_{0}: \theta=0$ the distribution of the $X_{j}$ is symmetric about zero. Let $0<Z_{1}<\cdots<Z_{N}$ denote the order statistics of $\left|X_{1}\right|, \ldots,\left|X_{N}\right|$. Introduce, for $j=1, \ldots, N$,

$$
V_{j}= \begin{cases}1 & \text { if the } X_{i} \text { corresponding to } Z_{j} \text { is }>0, \\ 0 & \text { otherwise. }\end{cases}
$$

Moreover, let $J$ be a continuous function on $(0,1)$ and let $U_{1: N}<\cdots<U_{N: N}$ be the order statistics of a sample of size $N$ from the uniform distribution on $(0,1)$. Then we introduce the exact scores

$$
a_{j}=a_{j N}=E J\left(U_{j: N}\right),
$$

$j=1, \ldots, N ; N=1,2, \ldots$ Then the one-sample linear rank statistic for testing $\mathrm{H}_{0}$ is given by

$$
T=\sum_{j=1}^{N} a_{j} V_{j}
$$

The appropriate norming constants (cf. (2.1)) are given by

$$
\zeta=E_{0} T=\frac{1}{2} \sum_{j=1}^{N} a_{j}, \quad \beta=\sigma_{0}(T)=\frac{1}{2}\left(\sum_{j=1}^{N} a_{j}^{2}\right)^{1 / 2} .
$$

An approximation $\tilde{G}(\tilde{x})$ for the $\mathrm{df} G(x)$ of the statistic $S$ thus obtained, both under $\mathrm{H}_{0}: \theta=0$ and under contiguous alternatives, is available to order $N^{-1}$ from Theorem 4.1 of ABZ. We shall now quote a version of this result. For brevity we shall be no more explicit than is strictly necessary for our present purpose. For full details, consult ABZ.

To begin with, we shall stipulate the conditions on $F$ and $J$. Let $\mathscr{Q}$ be the class of twice continuously differentiable functions $Q$ on $(0,1)$ that satisfy

$$
\limsup _{t \rightarrow 0.1} t(1-t)\left|\frac{Q^{\prime \prime}(t)}{Q^{\prime}(t)}\right|<\frac{3}{2}
$$

Let $\widetilde{\mathscr{F}_{1}}$ be the class of df's $F$ on $\mathbb{R}^{1}$ with positive densities that are symmetric about zero, four times differentiable, and such that, for $\psi_{i}=f^{(i)} / f, \Psi_{i}(t)=$ $\psi_{i}\left(F^{-1}((1+t) / 2)\right), m_{1}=6, m_{2}=3, m_{3}=4 / 3, m_{4}=1$, we have $\Psi_{1} \in \mathscr{Q}$ (cf. (3.5)) and 


$$
\limsup _{y \rightarrow 0} \int_{-\infty}^{\infty}\left|\psi_{i}(x+y)\right|^{m,} f(x) \mathrm{d} x<\infty, \quad i=1, \ldots, 4 .
$$

Let $\mathscr{Z}_{1}$ be the class of nonconstant functions $J$ on $(0,1)$ that satisfy $J \in \mathscr{Q}$ and $\int_{0}^{1} J^{4}(t) \mathrm{d} t<\infty$.

Next we introduce the approximation itself. In $\tilde{x}=x-\eta$, let

$$
\eta=-N^{1 / 2} \theta \frac{\int J \Psi_{1}}{\left(\int J^{2}\right)^{1 / 2}}
$$

where we use the convention that integration will be over $(0,1)$, unless stated otherwise. Moreover, let

where

$$
\tilde{G}(x)=\Phi(x)+\phi(x)\left\{\frac{1}{2} N^{-1} \eta \tilde{b_{0}}(N)+\sum_{k=0}^{3} N^{(1-k) / 2} \theta^{(3-k)} b_{k} H_{k}(x)\right\},
$$

in which

$$
\tilde{b}_{0}(N)=2 \sum_{i=1}^{N} \frac{\operatorname{cov}_{j}}{\int J \Psi_{1}}-\sum_{i=1}^{N} \frac{\sigma_{j}^{2}}{\int J^{2}}
$$

$$
\operatorname{cov}_{j}=\operatorname{cov}\left(J\left(U_{j: N}\right), \Psi_{1}\left(U_{j: N}\right)\right), \quad \sigma_{j}^{2}=\sigma^{2}\left(J\left(U_{j: N}\right)\right),
$$

and the $b_{k}, k=0,1,2,3$, are as given in $\tilde{K}_{\theta}(x)$ in (4.7) of $\mathrm{ABZ}$.

Here it suffices to know that these coefficients depend on $J$ and $F$, but not on $\theta$ and $N$. Maybe it is also useful to remark that the $b_{k}$ are explicit functions of $J$ and $F$ and hence that $\tilde{G}$ in $(3.8)$ is unique. Now we can formulate

Theorem 3.1. Let $F \in \tilde{F}_{1}, J \in \mathscr{A}_{1}$ and $0 \leqslant \theta \leqslant C N^{-1 / 2}$ for some $C>0$. For fixed $F$, $J$ and $C$ there exist positive numbers $\tilde{\delta}_{1}, \tilde{\delta}_{2}, \ldots$ such that $\lim _{N \rightarrow \infty} \tilde{\delta}_{N}=0$ and for every $N$,

$$
\sup _{x}|G(x)-\tilde{G}(\tilde{x})| \leqslant \tilde{\delta}_{N} N^{-1}
$$

Proof. This is a version of Theorem 4.1 from ABZ.

Now suppose again ou sample has been split into $r$ subsamples of sizes $N_{v}$, $v=1, \ldots, r$. For each of these samples we obtain a rank statistic $T_{v}$ as in (3.3), based on exact scores, which in analogy to (3.2) are defined by

$$
a_{j}(v)=a_{j: N_{1}}=E J\left(U_{j: N_{1}}\right) .
$$

I Ience $\beta_{v}^{2}-\sigma_{0}^{2}\left(T_{v}\right)-\sum_{j=1}^{N_{v}} a_{j}^{2}(v) / 2$ and the standardized version $S^{*}=\sum_{v=1}^{r} \gamma_{v} S_{v}$ of $T^{*}=\sum_{\nu=1}^{r} T_{v}$ is based on

$$
\gamma_{v}^{2}=\sum_{j=1}^{N_{v}} a_{j}^{2}(v) /\left(\sum_{g=1}^{r} \sum_{j=1}^{N_{2}} a_{j}^{2}(g)\right) .
$$

Moreover, in analogy to (3.9) and (3.10) we introduce

$$
\tilde{b_{0}}\left(N_{v}\right)=2 \sum_{j-1}^{N_{v}} \operatorname{cov}_{j}(v) / \int J \Psi_{1}-\sum_{j=1}^{N_{v}} \sigma_{j}^{2}(v) / \int J^{2},
$$


where $\operatorname{cov}_{j}(v)=\operatorname{cov}\left(J\left(U_{j: N_{r}}\right), \Psi_{1}\left(U_{j: N_{t}}\right)\right)$ and $\sigma_{j}^{2}(v)=\sigma^{2}\left(J\left(U_{j: N_{r}}\right)\right)$. Now we are in a position to formulate the main result of this section.

Theorem 3.2. Suppose that $\tilde{\gamma}_{1}^{2}=N_{1} / N \geqslant \varepsilon$ for some positive constant $\varepsilon$ and $v=$ $1, \ldots, r$. Then we have under the conditions of Theorem 3.1,

$$
\sup _{x}\left|G^{*}(x)-\tilde{G}(\tilde{x})-\phi(\tilde{x}) \frac{\eta}{2 N}\left\{\sum_{v-1}^{r} \tilde{b_{0}}\left(N_{v}\right)-\tilde{b_{0}}(N)\right\}\right| \leqslant \tilde{\delta_{N}} N^{-1} .
$$

Proof. Since $\tilde{\gamma}_{v}^{2} \geqslant \varepsilon$ for all $v$, Theorem 3.1 provides an expansion $\tilde{G}_{v}\left(x_{v}\right)$ for the $\mathrm{df}$ $G_{r}$ of the standardized version of $T_{r}, v=1, \ldots, r$. Hence Lemma 2.1 provides the approximation $\tilde{G}^{*}\left(x^{*}\right)$ from $(2.7)$ and $(2.8)$ for $G^{*}(x)$. As concerns the remainder in (2.7), we note in the first place that

$$
\sum_{1,=1}^{r} \delta_{r^{\prime}}=\sum_{r^{\prime}=1}^{r} \delta\left(N_{r^{\prime}}\right)=\sum_{r^{\prime}=1}^{r} \tilde{\delta}_{N} N_{r^{\prime}}{ }^{\prime} \leqslant(\varepsilon N)^{\prime} \sum_{r^{\prime}=1}^{r} \tilde{\delta}_{N_{r}}=\mathrm{o}\left(N^{-1}\right) .
$$

Moreover, according to ABZ (see p. 155), $N^{-1} \eta \tilde{b}_{o}(N)=\mathrm{o}\left(N^{-1 / 2}\right)$, whereas the remaining coefficients in (3.8) are clearly of order $N$ 1. Hence the other remainder terms in $(2.7)$ are $o\left(N^{-1}\right)$ as well.

Next we consider $\tilde{G}^{*}(x)$ itself. As each coefficient involved is $o\left(N^{-1 / 2}\right)$, the third and last part of $\tilde{G}^{*}(x)$ in $(2.8)$ is $o\left(N^{-1}\right)$ as well and thus can be relegated tot he remainder. For the middle part of $\tilde{G}^{*}(x)$ we resort to Lemma 2.2. The terms involving $N^{(1-k) / 2} \theta^{\left(3 \cdot k^{k}\right)} b_{k}$ in (3.8) satisfy (2.13) with $\gamma_{1}$, replaced by $\tilde{\gamma}_{1}$. As $\sum_{j=1}^{N} a_{j}^{2}=$ $N \int J^{2}-\sum_{j=1}^{N} \sigma_{j}^{2}$, it follows from (3.13) that

$$
\gamma_{j}-\tilde{\gamma}_{j}=\mathrm{O}\left(N^{-1} \sum_{j=1}^{N} \sigma_{j}^{2}\right)=\mathrm{o}\left(N^{-1 / 2}\right) .
$$

Hence $\bar{G}^{*}$ and $\tilde{G}$ may not agree exactly for these coefficients, but the replacement of $\gamma_{j}$ by $\tilde{\gamma}_{j}$ in terms of order $N^{1}$ in view of (3.16) leads to differences of o $\left(N^{-3 / 2}\right)$ which are amply negligible.

The only term which does cause a difference is the one involving $\tilde{b_{0}}(N)$ in $(3.8)$, which does not follow (2.13). Applying (2.8), we obtain that it leads in $\tilde{G}^{*}$ to a term involving

$$
\sum_{v=1}^{r} N_{v}^{-1} \eta_{v} \tilde{b_{0}}\left(N_{v}\right) \gamma_{v}=N^{-1} \eta \sum_{v^{\prime}=1}^{r} \tilde{b_{0}}\left(N_{v}\right) \gamma_{v} / \tilde{\gamma}_{v}=N^{-1} \eta \sum_{v=1}^{r} \tilde{b_{0}}\left(N_{v}\right)+\mathrm{o}\left(N^{-1}\right),
$$

using that $\eta_{v}=\tilde{\gamma}_{v} \eta$ and (3.16). This precisely accounts for the difference between (3.11) and (3.15).

However, there still remains onc point to be clcarcd. In (2.7), the approximation uses $x^{*}=x-\Sigma_{v=1}^{r} \gamma_{1} \eta_{v}$, while in (3.15) we simply use $\tilde{x}=x-\eta$. But

$$
\sum_{v=1}^{r} \gamma_{v} \eta_{v}=\eta \sum_{v=1}^{r} \gamma_{v} \tilde{\gamma}_{v}=\eta\left\{1-\frac{1}{2} \sum_{v=1}^{r}\left(\gamma_{v}-\tilde{\gamma}_{v}\right)^{2}\right\}=\eta+\mathrm{o}\left(N^{-1}\right)
$$

in view of (2.6), $(2.11)$ and $(3.16)$. 
The conclusion from Theorem 3.2 is that although the difference between the combined statistic $T^{*}$ and the total statistic $T$ no longer vanishes like in the trivial example from the previous section, it is still remarkably small and simple. To make this even more transparent, we shall translate the result into terms of powers and deficiencies. Let $\pi(\theta)$ denote the power of the level $\alpha$ test based on $T$ against $\mathbf{H}_{1}$ : $\theta>0$, and define $\pi^{*}(\theta)$ analogously. Recall that the deficiency $d_{N}$ of $T^{*}$ with respect to $T$ is the additional number of observations required by the test based on $T^{*}$ to match the performance with $T$ based on $N$ observations. Let $u_{\alpha}$ be such that $\alpha=1-\Phi\left(u_{\alpha}\right)$. Then we have

Corollary 3.1. Suppose that the conditions of Theorems 3.1 and 3.2 hold and that moreover $\bar{\varepsilon} \leqslant \alpha \leqslant 1-\tilde{\varepsilon}$ for some constant $\bar{\varepsilon}>0$. Then

$$
\begin{aligned}
& \pi(\theta)-\pi^{*}(\theta)=\phi\left(u_{\alpha}-\eta\right) \frac{\eta}{2 N}\left\{\sum_{v=1}^{1} \tilde{b}_{0}\left(N_{v}\right)-\tilde{b}_{0}(N)\right\}+o\left(N^{-1}\right), \\
& d_{N}=\sum_{v=1}^{r} \tilde{b}_{0}\left(N_{v}\right)-\tilde{b}_{0}(N)+\mathrm{o}(1) .
\end{aligned}
$$

Proof. Let $\xi_{\alpha}\left(\xi_{\alpha}^{*}\right)$ be the critical value of the test based on $T\left(T^{*}\right)$ at level $\alpha$. Note that (3.11) and (3.15) imply that $\xi_{\alpha}^{*}=\xi_{\alpha}+o\left(N^{-1}\right)$. Hence

$$
\pi(\theta)-\pi^{*}(\theta)=G^{*}\left(\xi_{\alpha}^{*}\right)-G\left(\xi_{\alpha}\right)=G^{*}\left(\xi_{\alpha}\right)-G\left(\xi_{\alpha}\right)+o\left(N^{-1}\right) .
$$

Another application of (3.11) and (3.15) and of the fact that $\xi_{\alpha}=u_{\alpha}+o(1)$, produces (3.17). As concerns $d_{N}^{\prime}$, we note that replacing $N$ by $N+d_{N}$ entails replacing $\eta$ by $\eta\left(1+N^{-1} d_{N}\right)^{1 / 2}$. Since the leading term in the expansions for both $\pi(\theta)$ and $\pi^{*}(\theta)$ equals $1-\Phi\left(u_{\alpha}-\eta\right)$, this replacement increases the power by $\phi\left(u_{\alpha}-\eta\right) \eta d_{N} /(2 N)+$ $\mathrm{o}\left(N^{-1}\right)$. Combination of this result with $(3.17)$ leads to $(3.18)$.

Hence, independent of the level $\alpha$ or the alternative $\theta$, the additional number of observations to be paid as a penalty for the splitting, equals to first order $\sum_{v^{\prime}=1}^{r} \tilde{b}_{0}\left(N_{v}\right)-\tilde{b}_{0}(N)$. Note that in the locally most powerful case $J=-\Phi_{1}$ we have from (3.14) and (3.9) that

$$
d_{N}=\left(\sum_{v=1}^{r} \sum_{j=1}^{N} \sigma_{j}^{2}(v)-\sum_{j=1}^{N} \sigma_{j}^{2}\right) / \int \Psi_{1}^{2}+o(1) .
$$

To close this section, we shall present some examples, parallelling those in ABZ. Hence we consider the one-sample normal scores (NS) and Wilcoxon (W) tests, against both normal (G) and logistic (L) alternatives. (We use $G$ from Gaussian as $\mathrm{N}$ from normal might lead to confusion with the sample size $N$.) For the computations involved, consult $\mathrm{ABZ}$ (and the correction note).

For the NS, we note that $\sum_{j=1}^{N} \sigma_{j}^{2}=\frac{1}{2} \log \log N+\frac{1}{2} \gamma+o(1)$, where $\gamma$ is Euler's constant given by $\gamma=\lim _{k \rightarrow \infty}\left(\sum_{i=1}^{k} i^{-1}-\log k\right)=0.577216 \ldots$ Hence (3.19) implies that 


$$
d_{N}(\mathrm{NS}, \mathrm{G})=\frac{1}{2} \sum_{r=1}^{r} \log \log N_{v}-\frac{1}{2} \log \log N+\frac{r-1}{2} \gamma+\mathrm{o}(1) .
$$

Since $\tilde{\gamma}_{v}^{2} \geqslant \varepsilon$, we also have $d_{N}(\mathrm{NS}, \mathrm{G})=\frac{1}{2}(r-1)(\log \log N+\gamma)+\mathrm{o}(1)$. Hence for each additional subgroup, we pay to first order $\frac{1}{2} \log \log N+\frac{1}{2} \gamma$ additional observations. As this quantity attains the values $\frac{1}{2}, 1,1 \frac{1}{2}$ and 2 for $N=4.6,63,7.9 \times 10^{4}$ and $2.0 \times 10^{13}$, respectively, it is clear that typically one additional observation will suffice. For some numerical rather than asymptotic approximations for $\sum_{j=1}^{N} \sigma_{j}^{2}$ also consult Albers (1974), p. 118.

For the second example of a locally most powerful test, we obtain simply

$$
d_{N}(\mathrm{~W}, \mathrm{~L}) \rightarrow d(\mathrm{~W}, \mathrm{~L})=\frac{1}{2}(r-1) .
$$

Hence one additional observation allows for two additional groups. For the remaining two mixed cases we find

$$
\begin{aligned}
d_{N}(\mathrm{~W}, \mathrm{G}) \rightarrow & d(\mathrm{~W}, \mathrm{G})=(\mathrm{r}-1)\left(\frac{7}{2}-2 \sqrt{2}\right)=(r-1) 0.671573 \ldots, \\
d_{N}(\mathrm{NS}, \mathrm{L})= & (r-1)\left(4-2 \sqrt{2}-\frac{\gamma}{2}\right) \\
& -\frac{1}{2}\left(\sum_{v=1}^{r} \log \log N_{v}-\log \log N\right)+\mathrm{O}(1),
\end{aligned}
$$

or equivalently, $d_{N}(\mathrm{NS}, \mathrm{L})=\frac{1}{2}(r-1)(1.765930-\log \log N)+\mathrm{o}(1)$. Note the remarkable phenomenon that the first order term in (3.23) can become negative. This probably is a reflection of the fact that the NS score function is slightly too steep for logistic alternatives, which effect is lessened by splitting. Also note, however, that this point is mainly of theoretical interest: even for $N_{1}=N_{2}=\frac{1}{2} N$, it takes $N=1295$ before the expression in (3.23) reaches 0 . And at e.g. $N=10^{4}$ it has only reached -0.15 .

\section{The two-sample case}

In this section we shall consider the two-sample case. The development will closely parallel that of the previous section. The situation will be considerably more complicated, however. This is due to the fact that here the underlying distribution is no longer supposed to be symmetric, which results in terms of order $N^{-1 / 2}$. Nevertheless, by applying the hitherto unused second part of Lemma 2.2, we shall be able to get rid of almost all terms in this case as well.

Let $X_{1}, \ldots, X_{m}$ be i.i.d. r.v.'s with common df $F$ and let $X_{m+1}, \ldots, X_{m+n}$ be i.i.d. r.v.'s with common df $F(x-\theta)$. Suppose that for some $\bar{\varepsilon}>0$,

$$
\bar{\varepsilon} \leqslant \lambda \leqslant 1-\bar{\varepsilon},
$$

where $\lambda=n / N$, with $N-m+n$. Let $Z_{1}<\cdots<Z_{N}$ now be the order statistics of 
$X_{1}, \ldots, X_{N}$, rather than of the absolute values. Moreover, no longer set $V_{j}=1$ in (3.1) if the $X_{i}$ corresponding to $Z_{j}$ is positive, but instead if it belongs to the second sample, that is, if $i \geqslant m+1$. Then the statistic $T$ in (3.3) has been adapted to testing the present hypothesis $\mathrm{H}_{0}: \theta=0$. Following $\mathrm{BZ}$, we shall assume without essential loss of generality that

$$
\sum_{j=1}^{N} a_{j}=0
$$

The appropriate norming constants (cf. (2.1) and (3.4)) now are $\zeta=E_{0} T=0, \beta=$ $\sigma_{0}(T)=\left(\lambda(1-\lambda) \sum_{j=1}^{N} a_{j}^{2}\right)^{1 / 2}$. The approximation $\tilde{G}(\tilde{x})$ for the df $G(x)$ of the statistic $S$ thus obtained, both under $\mathrm{H}_{0}$ and contiguous alternatives is available to order $N^{-1}$ from Theorem 5.1 of BZ. Again we shall be no more explicit than strictly necessary and refer to $\mathrm{BZ}$ for details.

Let $\mathscr{F}_{2}$ be the class $\mathscr{F}_{1}$ from Section 3 (see (3.6)) without the assumption of symmetry and with $\frac{1}{2}(1 \mid t)$ replaced by $t$ in $\Psi_{i}^{\prime}$. Likewise, let $\mathscr{Z}_{2}$ be the class $\mathscr{Z}_{1}$ with the additional assumption $\int J=0$ (cf. (4.2)) and with $\int J^{4}<\infty$ replaced by $\lim _{l \rightarrow 0,1}\{t(1-t)\}^{7 / 6} J^{\prime}(t)=0$. In $\tilde{x}=x-\eta$, let

$$
\eta=-(\lambda(1-\lambda) N)^{1 / 2} \theta \int J \Psi_{1} /\left(\int J^{2}\right)^{1 / 2}
$$

(cf. (3.7)), and define

$$
\tilde{G}(x)=\Phi(x)+\phi(x)\left\{\frac{1}{2} N^{-1} \eta \tilde{b_{0}}(N)+\sum_{k=0}^{5} b_{k}(\theta, N) H_{k}(x)\right\}
$$

(cf. (3.8)), where $\tilde{b_{0}}(N)$ is still given by (3.9) and

$$
\begin{aligned}
& b_{0}(\theta, N)=N^{1 / 2} \theta^{2} b_{0}^{(1)}+N^{-1 / 2} \theta b_{0}^{(2)}+N^{1 / 2} \theta^{3} b_{0}^{(3)}, \\
& b_{1}(\theta, N)=\theta b_{1}^{(1)}+\theta^{2} b_{1}^{(2)}+N \theta^{4} b_{1}^{(3)}+N^{-1} b_{1}^{(4)} \\
& b_{2}(\theta, N)=N^{-1 / 2} b_{2}^{(1)}+N^{-1 / 2} \theta b_{2}^{(2)}+N^{1 / 2} \theta^{3} b_{2}^{(3)} \\
& b_{3}(\theta, N)=N^{-1} b_{3}^{(1)}+\theta^{2} b_{3}^{(2)} \\
& b_{4}(\theta, N)=N^{-1 / 2} \theta b_{4}^{(1)} \\
& b_{5}(\theta, N)=N^{-1} b_{5}^{(1)}
\end{aligned}
$$

with the $b_{k}^{(j)}$ as given in $\bar{K}_{1}(x)$ from $(5.8)$ and (5.9) of BZ.

Theorem 4.1. Let $F \in \mathscr{F}_{2}, J \in \mathscr{Z}_{2}, 0 \leqslant \theta \leqslant C N^{-1 / 2}$ for some $C>0$. Under (4.1) there exist for fixed $F, J, C$ and $\bar{\varepsilon}$ positive numbers $\tilde{\delta}_{1}, \tilde{\delta}_{2}, \ldots$ such that $\lim _{N \rightarrow \infty} \tilde{\delta}_{N}=0$ and for every $N$

$$
\sup _{x}|G(x)-\tilde{G}(\tilde{x})| \leqslant \tilde{\delta}_{N} N^{-1} .
$$

Proof. This is a version of Theorem 5.1 from BZ. 
Next we again split $N$ into $\sum_{v=1}^{r} N_{v}$, observing the side-condition (1.1), which also implies that $n_{v} / N_{v}=\lambda, v=1, \ldots, r$. Then $\gamma_{v}$ is again given by (3.13) and we can formulate the main result of this section.

Theorem 4.2. Suppose that $\tilde{\gamma}_{v}^{2}=N_{v} / N \geqslant \varepsilon$ for some positive constant $\varepsilon$ and $v=1, \ldots, r$. Then we have, under the conditions of Theorem 4.1,

$$
\begin{aligned}
& \sup _{x}\left|G^{*}(x)-\tilde{G}(\tilde{x})-\frac{\phi(\tilde{x})}{2 N}\left\{\eta\left[\sum_{v=1}^{r} \tilde{b}_{0}\left(N_{v}\right)-\tilde{b}_{0}(N)\right]-(r-1)(\tilde{x}+2 \eta)\right\}\right| \\
& \quad \leqslant \tilde{\delta}_{N} N^{-1}
\end{aligned}
$$

Proof. Just as in Theorem 3.2 we begin by applying Lemma 2.1.

The remainder in (2.7) still is o $\left(N^{-1}\right)$ : for $\sum_{v=1}^{r} \delta_{v}$, the argument goes through without change, while for the other remainder terms the fact that the coefficients are now $O\left(N^{-1 / 2}\right)$ rather than $O\left(N^{-1 / 2}\right)$, still amply suffices. From (4.5) we learn that the three terms involving $b_{k}^{(1)}, k=0,1,2$, are of order $N^{-1 / 2}$, while the others are all of order $N^{-1}$. Hence the third and last part of $\tilde{G}^{*}(x)$ in (2.8) only plays a role as far as the six resulting combinations of these coefficients are concerned. All other contributions are o $\left(N^{-1}\right)$. Next we turn to the middle part of $\tilde{G}^{*}(x)$. Inspection of (4.5) reveals that the terms involving $b_{0}^{(1)}, b_{0}^{(3)}, b_{1}^{(1)}, b_{1}^{(2)}, b_{2}^{(1)}, b_{2}^{(2)}$, and $b_{3}^{(1)}$ satisfy (2.13) in Lemma 2.2, if $\gamma_{\nu}$ is replaced by $\tilde{\gamma}_{v}$. As all coefficients are $\mathrm{O}\left(N^{-1 / 2}\right)$, this in view of (3.16) leads to differences between $\tilde{G}$ and $\tilde{G}^{*}$ which are $o\left(N^{-1}\right)$, which is precisely tolerable as this is the order of accuracy of the expansions we are working with.

Next we turn to the second part of Lemma 2.2. Inspection of (5.8) in BZ shows that

$$
\begin{aligned}
& b_{1}^{(3)}=-\frac{1}{2}\left(b_{0}^{(1)}\right)^{2}, \quad b_{2}^{(3)}=-b_{0}^{(1)} b_{1}^{(1)}, \quad b_{3}^{(2)}=-\frac{1}{2}\left(b_{1}^{(1)}\right)^{2}-b_{0}^{(1)} b_{2}^{(1)}, \\
& b_{4}^{(1)}=-b_{1}^{(1)} b_{2}^{(1)} \quad \text { and } \quad b_{5}^{(1)}=-\frac{1}{2}\left(b_{2}^{(1)}\right)^{2} .
\end{aligned}
$$

It follows that $\tilde{G}$ and $\tilde{G}^{*}$ also agree to $o\left(N^{-1}\right)$ as far as these terms are concerned, and moreover that all six combination terms mentioned before have been accounted for.

Making up the balance in (4.5), we see that only $b_{0}^{(2)}$ and $b_{1}^{(4)}$ fall outside the scope of Lemma 2.2. Hence these terms we need to retrieve from (5.8) in BZ, obtaining that $N^{-1 / 2} \theta b_{0}^{(2)}=-\eta / N$ and $N^{-1} b_{1}^{(4)}=-1 /(2 N)$. Treating these terms just as $\tilde{b_{0}}(N)$, we arrive at (4.7). The argument according to which $x^{*}=\tilde{x}+\mathrm{o}\left(N^{-1}\right)$ goes through unchanged, which concludes the proof.

Comparing Theorem 4.2 to Theorem 3.2, we observe that although the twosample expansion given by (4.4) and (4.5) is much more complicated than its onesample counterpart in (3.8), the resulting increase of discrepancy between $G^{*}$ and $G$ is again surprisingly small: the only difference between (4.7) and (3.15) is the $(r-1)(\tilde{x}+2 \eta)$-term. 
In the corollary below the result is reformulated in terms of powers and deficiencies.

Corollary 4.1. Suppose that the conditions of Theorems 4.1 and 4.2 hold and that moreover $\bar{\varepsilon} \leqslant \alpha \leqslant 1-\bar{\varepsilon}$ for some constant $\bar{\varepsilon}>0$. Then

$$
\begin{aligned}
& \pi(\theta)-\pi^{*}(\theta)= \phi\left(u_{\alpha}-\eta\right) \frac{\eta}{2 N}\left\{\sum_{v=1}^{r} \tilde{b}_{0}\left(N_{v}\right)-\tilde{b}_{0}(N)-(r-1)\right\} \\
&+\mathrm{o}\left(N^{-1}\right), \\
& d_{N}=\sum_{v=1}^{r} \tilde{b}_{0}\left(N_{v}\right)-\tilde{b}_{0}(N)-(r-1)+\mathrm{o}(1) .
\end{aligned}
$$

Proof. From (4.6) and (4.7) it follows that the critical values satisfy $\xi_{\alpha}^{*}=$ $\xi_{\ell}+(r-1) \xi_{r \alpha} /(2 N)+o\left(N^{-1}\right)$. Hence

$$
\begin{aligned}
\pi(\theta)-\pi^{*}(\theta) & =G^{*}\left(\xi_{\alpha}^{*}\right)-G\left(\xi_{\alpha}\right) \\
& =G^{*}\left(\xi_{\alpha}\right)-G\left(\xi_{\alpha}\right)+\phi\left(\xi_{\alpha}-\eta\right)(r-1) \xi_{\alpha} /(2 N)+o\left(N^{-1}\right) .
\end{aligned}
$$

Another application of (4.7) and of the fact that $\xi_{\alpha}=u_{\alpha}+o(1)$, leads to (4.8). The step from (4.8) to (4.9) is identical to that from (3.17) to $(3.18)$.

To conclude this section, we present the results parallelling (3.20)-(3.23) for the two-sample case. Note that the passage fion (3.18) to (4.9) is not just a matter of subtracting $(r-1)$ from the first result to obtain the latter: in going from $\mathscr{F}_{1}$ to $\mathscr{F}_{2}$, we replace $\frac{1}{2}(1+t)$ in $\Psi_{\mathrm{I}}$ by $t$. We obtain subsequently

$$
\begin{aligned}
& d_{N}(\mathrm{NS}, \mathrm{G})=\sum_{v=1}^{r} \log \log N_{v}-\log \log N+(r-1)(\log 2+\gamma-1)+\mathrm{o}(1), \\
& d_{N}(\mathrm{~W}, \mathrm{~L}) \rightarrow d(\mathrm{~W}, \mathrm{~L})=(r-1) \\
& d_{N}(\mathrm{~W}, \mathrm{G}) \rightarrow d(\mathrm{~W}, \mathrm{G})-(r-1) \\
& d_{N}(\mathrm{NS}, \mathrm{L})=(r-1)(3-\log 2-\gamma)-\sum_{v=1}^{r} \log \log N_{v}+\log \log N+\mathrm{o}(1)
\end{aligned}
$$

Again some simplification can be achieved by replacing

$$
\sum_{v=1}^{r} \log \log N_{v}-\log \log N \text { by }(r-1) \log \log N
$$

in (4.10) and (4.13). A final remark is that in both the one- and the two-sample case we have

$$
d_{N}(\mathrm{NS}, \mathrm{G})=(r-1)\left(\tilde{d}_{N}(\mathrm{NS}, t)+\frac{1}{2}\right)+o(1)
$$

where $\tilde{d}_{N}(\mathrm{NS}, t)$ is the deficiency of the normal scores test with respect to the $t$-test (cf. Albers (1974), p. 122 and BZ, p. 989). 


\section{A numerical example}

By way of illustration we shall perform some simulations for the case of Wilcoxon's one-sample test against logistic location alternatives. We shall consider total sample sizes $N$ between 20 and 50 and numbers of subgroups $r$ between 2 and 5 , thus producing configurations $\left(N_{1}, \ldots, N_{r}\right)$ like $(10,10),(10,40),(20,10,10)$, $(10,10,10,10,10)$, etc. For $\alpha$ we choose 0.05 , while we typically select the shift $\theta$ in such a way that the power $\pi(\theta)$ is in the region of practical interest, say $(0.6,0.9)$.

Basically, the simulation method is of extreme simplicity: at each step, we draw a sample of size $N$ from the standard logistic df $F(x)=\left(1 \mid \mathrm{c}^{-x}\right)^{-1}$, shift it over $\theta$ and compute the Wilcoxon statistics $T, T_{1}, \ldots, T$, and $T^{*}$. The fractions of times in which $T$ and $T^{*}$ exceed their respective critical values are the desired estimators for $\pi(\theta)$ and $\pi^{*}(\theta)$, respectively. However, since we are interested in differences between powers and between sample sizes required, rather than in these quantities themselves, the relative errors in the simulation estimates tend to be quite large, even for numbers of simulations NS of order $10^{4}$.

Therefore, some refinements of the basic technique are applied, on which we shall now briefly comment. In the first place, the variance reducing technique of the antithetic variables is used: after each generation of a sample $X_{1}, \ldots, X_{N}$, we also utilize its counterpart $2 \theta-X_{1}, \ldots, 2 \theta-X_{N}$. Moreover, as we are interested in relatively small quantities, we should take advantage of this situation by estimating small probabilities, which will further decrease the variance. To be more specific, we estimate $\pi(\theta)-\pi^{*}(\theta)$ by

$$
\hat{\pi}_{10}-\hat{\pi}_{01} \text {, }
$$

where $\hat{\pi}_{10}$ is the fraction of times the ordinary test rejects but the combined one does not, and $\hat{\pi}_{01}$ is just the opposite.

Another complication which calls for some refinement, is the following. The estimators in (5.1) are obtained by counting the numbers of times the critical values are exceeded. For $T$, exact critical values are available from tables, but for $T^{*}$ this is obviously not the case. Now critical values for $T^{*}$ can be derived from those for $T$ along the following lines. According to Corollary 3.1, the standardized critical values $\xi_{\alpha}^{*}$ and $\xi_{\alpha}$ agree to $o\left(N^{-1}\right)$. The unstandardized critical values are obtained through multiplication of $\xi_{\alpha}^{*}$ by $\left(\sum_{r=1}^{r} \sum_{j=1}^{N_{v}} a_{j}^{2}(v)\right)^{1 / 2}$ and of $\xi_{\alpha}$ by $\left(\sum_{j=1}^{N} a_{j}^{2}\right)^{1 / 2}$. For $a_{j}=j /(N+1)$, the ratio of these factors equals

$$
1-\frac{r-1}{4 N}
$$

to $\mathrm{O}\left(N^{2}\right)$. Consequently, we shall use as critical values for $T^{*}$ those for $T$, after multiplication by the factor in (5.2).

The fact that the critical values for $T^{*}$ are not exact, calls for a corrected version of the estimator in (5.1). For inexact critical values, we really estimate some $\tilde{\pi}(\theta)$ and $\tilde{\pi}^{*}(0)$ rather than the intended $\pi(\theta)$ and $\pi *(\theta)$, respectively. Let $\tilde{\alpha}$ and $\tilde{\alpha}^{*}$ be 
the corresponding realized exceedance probabilities under $\mathrm{H}_{0}$, then it is easily verified that to first order

$$
\pi(\theta)-\pi^{*}(\theta)=\tilde{\pi}(\theta)-\tilde{\pi}^{*}(\theta)-C\left(\tilde{\alpha}-\tilde{\alpha}^{*}\right),
$$

in which $C=\phi\left(u_{x x}-\eta\right) / \phi\left(u_{x x}\right)$, with $\eta=(N / 3)^{1 / 2} \theta$. Combination of (5.1) and (5.3) now leads to the corrected estimator

$$
S=\left(\hat{\pi}_{10}-\hat{\pi}_{01}\right)-C\left(\hat{\alpha}_{10}-\hat{\alpha}_{01}\right)
$$

where $\hat{\alpha}_{10}$ and $\hat{\alpha}_{01}$ are defined as $\hat{\pi}_{10}$ and $\hat{\pi}_{01}$ but applied to each generated logistic sample before it is shifted over $\theta$.

The variance $\sigma^{2}(S)$ of $S$ is in itself rather complicated, but the simulations show that most of the terms involved are negligible, as expected. A suitable estimator for $\sigma^{2}(S)$ is

$$
\hat{\sigma}^{2}(S)=\left\{\left(\hat{\pi}_{10}+\hat{\pi}_{01}\right)+C^{2}\left(\hat{\alpha}_{10}+\hat{\alpha}_{01}\right)\right\} / N S .
$$

Note that the replacement of $(5.1)$ by (5.4) reduces the bias, but, in view of $C^{2}$ in (5.5), increases the variance.

Next we turn to the deficiency. From (3.17) and (3.18) we recall that $d_{N}$ and $\pi(\theta)-\pi^{*}(\theta)$ differ by a factor $\eta \phi\left(u_{\alpha}-\eta\right) /(2 N)$ to first order for $a_{j}=j /(N+1)$. Hence the obvious estimator for $d_{N}$ is

$$
\hat{d}_{N}=\frac{2(3 N)^{1 / 2} S}{\theta \phi\left(u_{\alpha}-(N / 3)^{1 / 2} \theta\right)}=\frac{2(3 N)^{1 / 2}}{\theta}-\left\{\frac{\left(\hat{\pi}_{10}-\hat{\pi}_{01}\right)}{\phi\left(u_{\alpha}-(N / 3)^{1 / 2} \theta\right)}-\frac{\left(\hat{\alpha}_{10}-\hat{\alpha}_{01}\right)}{\phi\left(u_{\alpha}\right)}\right\} .
$$

Also obviously, we find that $\hat{\sigma}\left(\hat{d}_{N}\right)=2(3 N)^{1 / 2} \hat{\sigma}(S) /\left(\theta \gamma\left(u_{\alpha}-(N / 3)^{1 / 2} \theta\right)\right)$.

A simulation study was carried out along the lines indicated above, using 40000 simulations for each case. On the basis of these simulations, the realized exceedance probabilities $\tilde{\alpha}^{*}$ (cf. (5.3)) turn out to behave quite satisfactorily. Their estimated values differ only marginally from those for $\bar{\alpha}$. They tend to be on the negative, and hence conservative, side, leading to an average of $4.9 \%$ for $\alpha=5 \%$.

For brevity we shall present the results for one typical example in detail, and give a summary table for the remaining cases. For $\alpha=0.05, N=40, r=3, N_{1}=N_{2}=10$, $N_{3}=20$ and $\theta=0.75$ we obtain $\hat{\alpha}_{11}=4.36 \%, \hat{\alpha}_{10}=0.61 \%, \hat{\alpha}_{01}=0.53 \%, \hat{\pi}_{11}=82.4 \%$, $\hat{\pi}_{10}=1.93 \%$ and $\hat{\pi}_{01}=1.12 \%$. Hence the estimates for $\pi(\theta), \alpha$ and $\alpha^{*}$ are respectively $84.3 \%, 4.97 \%$ and $4.89 \%$. As $C=2.13$ here, we obtain through (5.4)-(5.6) that the estimated power difference $S=0.65 \%$, with $\hat{\sigma}(S)=0.14 \%$, while the cstimated deficiency $\hat{d}_{N}=0.86$, with $\hat{\sigma}\left(\hat{d}_{N}\right)=0.14$. In Table 1 we list the results for $\pi(\theta), \hat{d}_{n}$ and $\hat{\sigma}\left(\hat{d}_{N}\right)$ for all configurations considered. The conclusion from Table 1 is rather straightforward: the estimated deficiencies show an agreement with the theoretical values $d=\frac{1}{2}(r-1)$ from (3.21) which in view of the estimated standard deviations is very satisfactory. For $r=2,3,4,5$, the average deficiencies obtained differ from $\frac{1}{2}(r-1)$ by $-0.08,+0.01,+0.07$ and -0.04 , respectively. 
Table 1

The power $\pi(\theta)$ (in $0_{0}$ ) of Wilcoxon's one-sample test against logistic location alternatives with shift $\theta$, the deficiency $\vec{d}_{N}$ with respect to this test of its combined counterpart, and the standard deviation $\hat{\sigma}\left(\bar{d}_{N}\right)$, all for level $\alpha=0.05$ and based on 40000 simulations

\begin{tabular}{|c|c|c|c|c|c|}
\hline$r$ & ${ }_{10}^{1}\left(N, \ldots, N_{,}\right)$ & $\theta$ & $\pi(\theta)$ & $\vec{d}_{x}$ & $\hat{\sigma}\left(\hat{d}_{N}\right)$ \\
\hline \multirow[t]{11}{*}{2} & $(1,1)$ & $!$ & 33.9 & 0.45 & 0.18 \\
\hline & $(1,1)$ & 1 & 78.4 & 0.69 & 0.10 \\
\hline & $(1,1)$ & 1 & 78.5 & 0.58 & 0.10 \\
\hline & $(1,2)$ & 1 & 91.3 & 0.58 & 0.13 \\
\hline & $(1,3)$ & l & 96.9 & 0.62 & 0.19 \\
\hline & $(2,2)$ & $\frac{1}{2}$ & 55.3 & 0.12 & 0.21 \\
\hline & $(2,2)$ & 1 & 96.8 & 0.51 & 0.19 \\
\hline & $(1,4)$ & $\frac{1}{2}$ & 63.8 & 0.30 & 0.23 \\
\hline & $(1,4)$ & $\frac{3}{4}$ & 90.7 & 0.19 & 0.18 \\
\hline & $(2,3)$ & $\frac{1}{2}$ & 64.0 & 0.39 & 0.22 \\
\hline & $(2,3)$ & 4 & 91.0 & 0.24 & 0.19 \\
\hline \multirow[t]{7}{*}{3} & $(1,1,1)$ & $\frac{3}{4}$ & 73.4 & 0.95 & 0.16 \\
\hline & $(1,1,1)$ & 1 & 91.3 & 1.01 & 0.16 \\
\hline & $(1,1,2)$ & $\frac{3}{4}$ & 84.3 & 0.86 & 0.19 \\
\hline & $(1,1,3)$ & $\frac{1}{3}$ & 63.9 & 0.98 & 0.27 \\
\hline & $(1,1,3)$ & $\frac{3}{4}$ & 91.0 & 1.16 & 0.23 \\
\hline & $(1,2,2)$ & $\frac{1}{5}$ & 63.9 & 0.72 & 0.28 \\
\hline & $(1,2,2)$ & 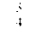 & 90.9 & 1.37 & 0.22 \\
\hline \multirow[t]{3}{*}{4} & $(1,1,1,1)$ & $\stackrel{\grave{i}}{i}$ & 84.2 & 1.59 & 0.22 \\
\hline & $(1,1,1,2)$ & $\frac{1}{3}$ & 63.8 & 1.25 & 0.31 \\
\hline & $(1,1,1,2)$ & $\frac{3}{4}$ & 90.9 & 1.86 & 0.25 \\
\hline \multirow[t]{2}{*}{5} & $(1,1,1,1,1)$ & $!$ & 63.9 & 1.90 & 0.33 \\
\hline & $(1,1,1,1,1)$ & $\frac{3}{4}$ & 90.8 & 2.02 & 0.27 \\
\hline
\end{tabular}

\section{Acknowledgement}

The author thanks Mrs. M. de Leeuw for carrying out the computations leading to Table 1 and a referee for detailed comments which helped to improve the presentation.

\section{References}

Albers, W. (1974). Asymptotic Expansions and the Deficiency Concept in Statistics. Math. Centre Tracts 58, Amsterdam.

Albers, W. (1991). Second order analysis of two-stage rank tests for the one-sample problem. Ann. Statist. 19, 1042-1052.

Albers, W. (1992). Asymptotic cxpansions for two-stage rank tests. To appear in Ann. Inst. of Statist. Math. 44.

Albers, W. and M.G. Akritas (1987). Combined rank tests for the two-sample problem with randomly censored data. J. Amer. Statist. Assoc. 82, 648-655. 
Albers, W., P.J. Bickel and W.R. van Zwet (1976). Asymptotic expansions for the power of distributionfree tests in the one-sample problem. Ann. Statist. 4, 108-156. Correction note: Ann. Statist. 6, $1170-1171$.

Bickel, P.J. and W.R. van Zwet (1978). Asymptotic expansions for the power of distribution-free tests in the two-sample problem. Ann. Statist. 6, 937-1004.

Cramér, H. (1946). Mathematical Methods of Stastistics. Princeton University Press, Princeton, NJ. Hodges, J.L. Jr. and E.L. Lehmann (1970). Deficiency. Ann. Statist. 41, 783-801.

Lehmann, E.L. (1975). Nonparametrics: Statistical Methods Based on Ranks. Holden Day, San Francisco, CA.

Widder, D.V. (1966). The Laplace Transform. 7th Print, Princeton Math. Series no, 6. Princeton University Press, Princeton, NJ.

van Zwet, W.R. and J. Oosterhoff (1967). On the combination of independent test statistics. Ann. Math. Statist. 38, 659-680. 